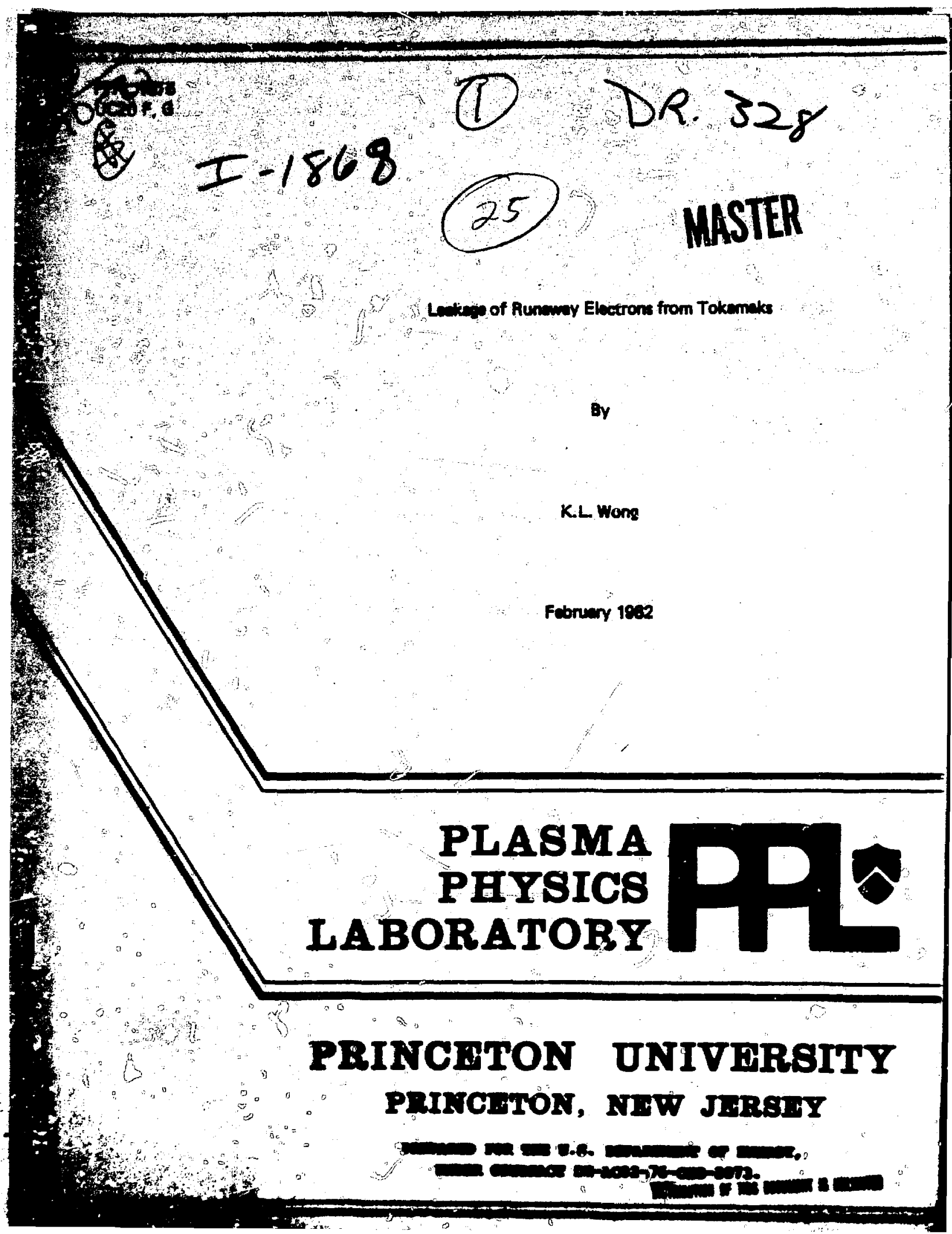




\title{
Leakage of Runaway Electrons From Tokamaks
}

\author{
R. I. Wong \\ Plasma Physics Laboratory, Princeton University \\ Princeton, New Jersey 08544
}

\section{ABSTRACT}

Runaway electron orbits are calculated : $x$ a tokamak magnetic field. It is shown that these elect ons tend to . drift towards a larger major radius with a veijcity $\vec{v}_{R}=$ $q C E / B_{O} \hat{R}$. This effect may be relevant to some recent experimental observations in tokamaks.

PPPL --1875

DE82 008317 
Runaway electrons with energies in MeV ronge have been observed in many tokamak discharges. ${ }^{1}$ These electrons mainly. interact with the electric and magnetic fields because the plasma frictional drag on these particles is very small. The transport of these electrons has been experimentally investigated in large tokamaks $^{2}$ (PLT and PDX), in search of some mechanisin which is related to the anomalous transport of the thermal electrons. In this note, we calculate the runaway electron orbits and show that they do not have closed drift surfaces, but rather tend to drift towards a larger major radius during acceleration in the ohmic electric field. The drift velocity is shown to be proportional to the accelerating electric field. This finding offers a possible explanation for the rapid reduction of hard x-ray emission observed in several lower hybrid wave current drive experiments in tokamaks. $3,4,5$

Consider a runaway electron with parallel (with respect to -the magnetic field) velocity $v_{n}$ much larger than che perpendicular velocity $v_{1}$ so that $v=\left(v_{n}^{2}+v_{\perp}^{2}\right)=v_{n}$. The electron will arift across the curved magnetic field line with a drift velocity

$$
\vec{v}_{d}=\frac{\gamma m_{0} c}{e B} \frac{v^{2}}{R} \frac{\vec{B} \times \vec{R}}{|\vec{B} \times \vec{R}|}=r \frac{m_{0} c}{e_{0}} \frac{v^{2}}{R_{0}} \frac{\vec{B} \times \vec{R}}{|\vec{B} \times \vec{R}|}
$$

where

$$
\begin{aligned}
& m_{0}=\text { electron rest mass } \\
& c=\text { speed of light }
\end{aligned}
$$




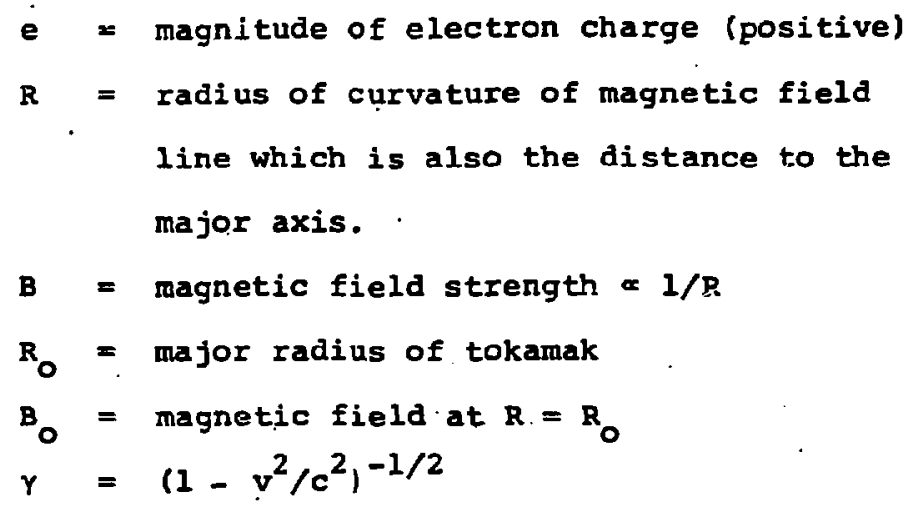

The electron orbit does not stay on the magnetic surface due to this curvature drift. Figure 1 shows the projection of the electron orbit onto a fixed toroidal plans. For simplicity, let us assume conceniric circular magnetic surfaces. Deviation from this assumption woula introduce minor quantitative differences. The electron starting at point $A\left(\theta=0, r=r_{0}\right)$ dififs towards a smaller minor radius $r$ when it is at the upper-half-plane. When it gets to the lower-half-plane, it starts to drift towards a larger $r$. As a zeroth order approximation, the electron moveis on the circular drift surface with its certer shifted outward by a distance $\Delta_{0} / 2$ with respect to the center of the magnetic surface. $^{l}$ Since the electron is accelerated by an electric field $E$ and the drift velocity $v_{d} \propto \gamma v^{2}$, one would expect the electron to drift a longer distance at the lower-half-plane despite the fact that it spends less time there $\left(t=v^{-1}\right)$. As a consequence, the electron will and up at point $B\left(\theta=0, r=r_{0}+\Delta_{1}\right)$ with $\Delta_{1}>0$. This mechanism bears some similarity with the wellknown Ware-pinch ${ }^{6}$ effect on trapped particles. Both of them are 
caused by the toroidal electric field. Fo calculate $\Delta_{1}$, let us write the radial component of the drift velocity as $\vec{v}_{r}=-v_{d}$ $\sin \theta \hat{r}$

and $\Delta_{1}=\int_{0}^{T} v_{r} d t=-\int_{0}^{T} v_{d} \sin \theta d t$,

where $T$ is the time it takes to go from point A to pojint $B$. Let $s$ denote the distance travelled by the electron. Ising the large-aspect-ratio approximation,

$$
\begin{aligned}
& s=\theta\left(R_{0}{ }^{2} q^{2}+r^{2}\right)^{1 / 2}=R_{0} q \theta \\
& \frac{d \theta}{d t}=\frac{d \theta}{d s} \frac{d s}{d t}=\frac{1}{R_{0} q} v_{,}
\end{aligned}
$$

so that

$$
d t=\frac{R_{0} q}{v} d \theta,
$$

where $q$ is the safety factor. Substituting Eq. (3) into Eq. (1), - we get

$$
\begin{aligned}
\Delta_{1} & =-\int_{0}^{2 \pi} v_{d} \sin \theta \cdot \frac{R_{G} q}{v} d \theta \\
& =-\int_{0}^{2 \pi} \frac{m_{0} c}{e_{0}} q r v \sin \theta d \theta .
\end{aligned}
$$

Let $r=r_{0}, v=v_{0}$ at $\theta=0$, from energy conservation, we have

$$
\begin{aligned}
\gamma m_{0} c^{2} & =r_{0} m_{0} c^{2}+e E R_{0} q \theta \\
r & =r_{0}+\frac{e E R_{0} q}{m_{0} c^{2}} \theta
\end{aligned}
$$


$-5-$

Therefore

$$
\begin{aligned}
\gamma v & =\gamma c\left(1-\gamma^{-2}\right)^{1 / 2} \\
& =c\left(\gamma^{2}-1\right)^{1 / 2} \\
& =\gamma_{0} v_{0}+\frac{e E R_{0} g \theta}{m_{0} v_{0}} .
\end{aligned}
$$

Obviously., aE $R_{0} q \theta / m_{0} v_{0} \ll \gamma_{0} v_{0}$ because the electron energy is much higher than the energy gain per transit around the torus. Substitute Eq. (5) into Eq. (4), we get

$$
\begin{aligned}
& \Delta_{1}=-\int_{0}^{2 \pi} \frac{m_{0} c}{e B_{0}} q\left(\gamma_{0} v_{0}+\frac{e E}{m_{0}} \frac{q R_{0}^{\theta}}{v_{0}}\right) \sin \theta d \theta \\
& \quad=2 \pi q^{2} R_{0} \frac{c}{v_{0}} \frac{E}{B_{0}} .
\end{aligned}
$$

similarly, we can calculate $\Delta_{0}$ by

$$
\begin{aligned}
\Delta_{0} & =-\int_{0}^{\pi} v_{d} \sin \theta \frac{R_{0} q}{v} d \theta \\
& =-2 \frac{m_{0} c}{e B_{0}} q Y_{0} v_{0} .
\end{aligned}
$$

Although $\left|\Delta_{1} / \Delta_{0}\right|=\pi \mathrm{eE} \mathrm{R}_{0} q / \gamma_{0} \mathrm{~m}_{0} v_{0}^{2} \ll 1$, there is a consistent tendency for the electron to drift outward along the major radius. with a velocity

$$
\begin{aligned}
\dot{v}_{R} & =\frac{\Delta_{1}}{I} \hat{R} \\
& =\Delta_{1} /\left(2 \pi R_{0} q / v_{0}\right) \hat{R} \\
& =q c E / B_{0} \hat{R} .
\end{aligned}
$$


It should be noted that the above calculation is valid only if the runaway electron stays close to the circular magnetic surface, i.e., $\Delta_{0} \ll \mathbf{r}_{0}$. Very energetic runaways can have $\Delta_{0}$ comparable to $r_{0}$. In fact, putting $\Delta_{0}$ equals half the limiter radius can give a reasonable estimate on the maximum energy of runaway electrons that can exist in a tokamak. In addition to the outward drift $\overrightarrow{\vec{v}}_{R}$ ' there is an $\vec{E} \dot{\mathrm{x}} \overrightarrow{\mathrm{B}}$ ' $\mathrm{dri}$ it pinching towards the minor axis with the velocity

$$
\vec{v}_{E}=\frac{\vec{E} \times \vec{B}}{B^{2}} c=-\frac{E\left|B_{\theta}\right|}{B^{2}} c \hat{r},
$$

which is less than $\vec{v}_{R}$ by the factor $\left|B_{\theta}\right| / q B \ll 1$ and therefore can be neglected.

The purpose of this calculation is to suggest an explanation for some recent observations in lower hybrid wave current arive experiments in tokamaks. 3,4,5 shortly after the injection of $R F$ power, the loop voltage drops rapidly because part of the ohmic heating current originally carried by the thermal electrons is transferred to the energetic, collisionless electrons that resonate with the wave. Associated with the loop voltage change is the simultaneous drop $\left(t_{x}-t_{v}\right)$ in the hard $x$-ray emission as shown in Fig. 2. The hard $x$-ray emission is expected to be porportional to the flux of runaway electrons $\Gamma$ hitting the limiter:

$$
\Gamma=n_{Y} v
$$


Obviously a decrease in loop voltage will slow down the runaway production rate, but the runaway electron density ny can only change in time scale of its confinement time which is significantly longer than $t_{x}$. This implies that the velocity $v$ in Eq. (10) is changing rapidly during the RF injection. It should be emphasized here that the outward drift $\vec{v}_{R}$ may not be the dominant transport mebcnaism for runaway electrons in tokamaks; in fact, there are strong evidence that magnetic field fluctuations can play an important role. ${ }^{2}$ However, previous experiments ${ }^{I}$ show that in low densjty discharges in $=1.2 \times$ $10^{13} \mathrm{~cm}^{-3}$ ), the runaway electrons are better confined and the orbit-shift model correctly predicts many of their properties. It is well-known that the lower hybrid wave current drive experiments $3,4,5$ are successful only in the low density (n $8 \times \times 10^{12}$. $\mathrm{cm}^{-3}$ ) slideaway regime. If the convective outward drift of the rur. way electrons is the dominant transport mechanism, at least in the vicinity of the limiter, then $V=v_{R} \propto \mathrm{E} \approx$. loop voltage which would explain the data shown in Fig. 2. The improvement of runaway confinement at low electric field also suggests the possibility of maintaining a low density, low loop voltage tokamak discharge for a long period of time as has been achieved recently in the ASDEX tokamak. 7

The author wishes to thank Dr. R. Kulsrud, Dr. P. Rutherford and Dr. J. Strachan for helpful discussions. Special thanks are due to Dr. T. H. Stix who corrected an algebraic error in the first draft of this manuscript. The work was supported by the U.S. Dept. of Energy Contract No. DE-ACO2-76-CHo3073. 


\section{REFERENCES}

$I_{H}$. Knoepfel and S. J. Zweben, Phys. Rev. Lett. 35, 1340 (1975).

${ }^{2}$ C. Barnes, Ph.D. Thesis, PPPL, Princeton University (1981).

3T. Yamamoto, T. Jmai, M. Shimada, N. Suzuki, M. Maeno,

S. Konoshima, T. Jujii, K. Uehara, T. Nagashima, A. Funahashi,

N. Fujisawa, Phys. Rev. Lett. 45, 716 (1980).

${ }^{4}$ s. C. Luckhardt, M. Porkolab, S. F. Knowlton, K-I. Chen,

A. S. Fisher, F. S. MeDermott, M. Mayberry, PFC/JA-81-14, Plasma Fusion Center, M.I.T.

$5_{W}$. Hooke, C. Barnes, S. Bernabei, C. Daughney, P. Efthimion, J. Hosea, D. Ignat, F. Jobes, R. Motley, J. Stevens, J. Strachan, Bull. Am. Phys. Soc. 26, 975 (1981).

6. A. Ware, Phys. Rev. Lett. 25 (1970).

${ }^{7}$ G. Fussmann et al., Phys. Rev. Lett. 47, 1004 (1981). 


\section{FTGURE CAPTIONS}

Fig. 1. Projection of the electron orbit on to a toroidal plane. An accelerating electron starts at point "A" arrives at point " $B$ " after one revolution around the magnetic axis.

Fig. 2. General features of the temporal evolution of (a) injected RF power, (b) toroidal loop voltage and (c) hard $x-r a y$ emission in typical lower hybrid wave current arive experiments (see references 3,4 and 5 ). 
Major

Axis

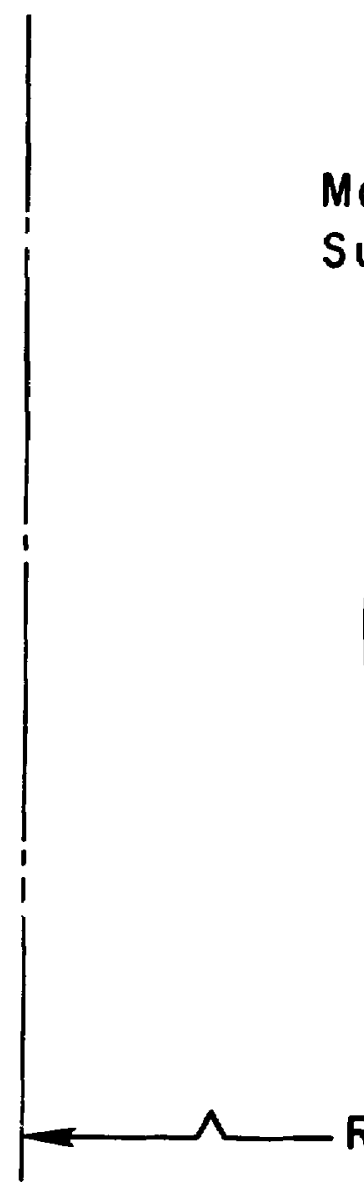

\# $81 \times 1422$

Electron

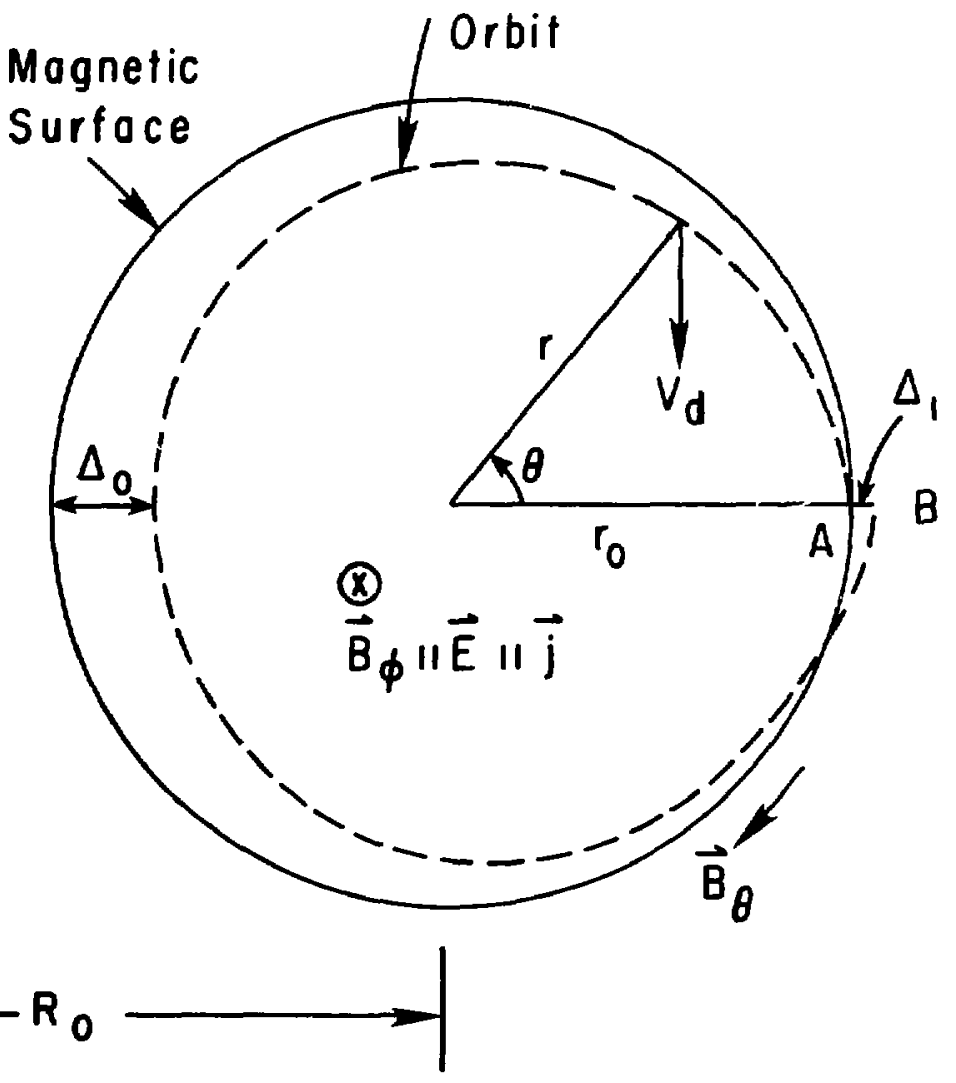

Fig. 1 

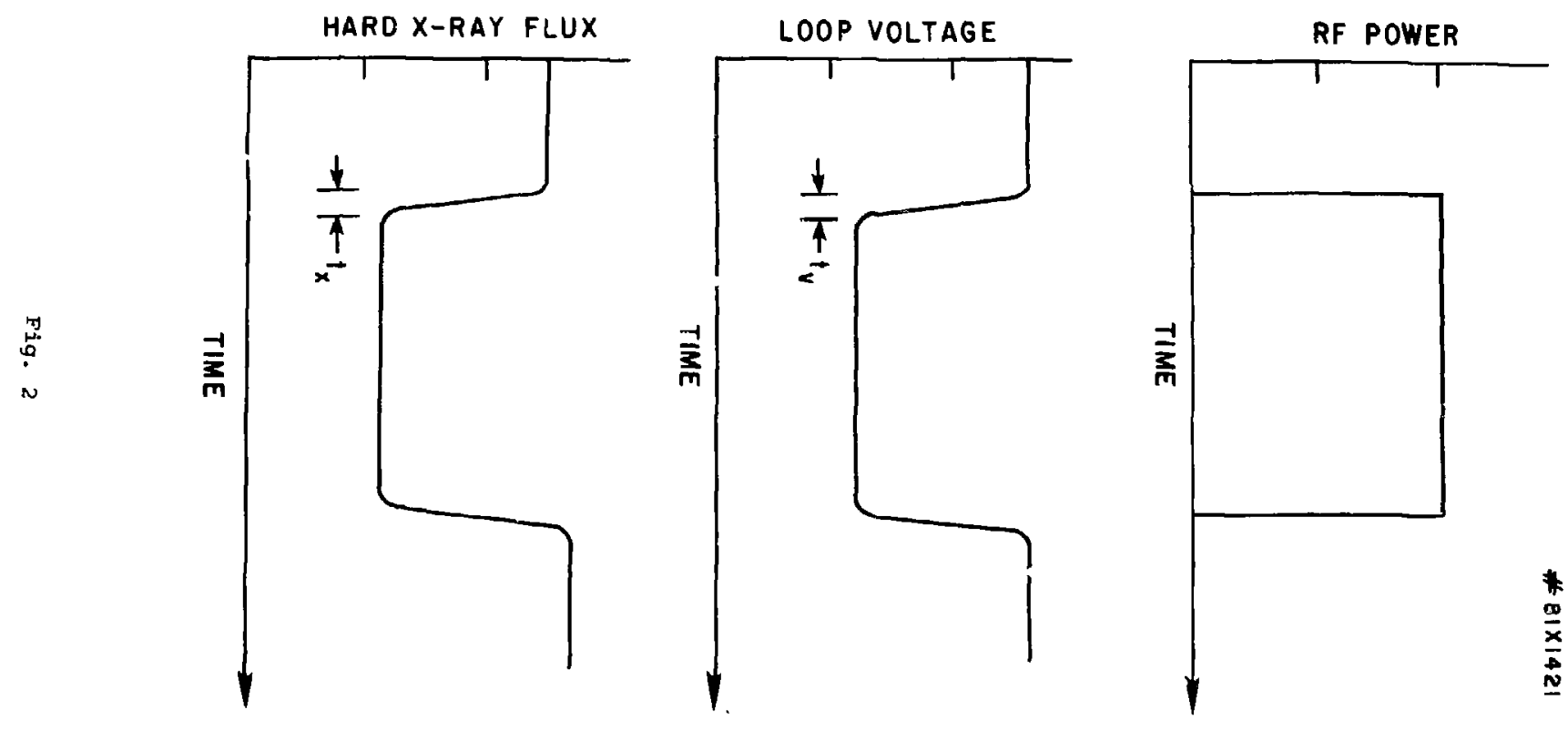